\title{
Adaptive MMSE Equalizer Based On CSLMS For Blind Fractional Spaced CMA Channel Equalization
}

\author{
R. Nirmala Devi, Prof. K.Kishan Rao
}

\begin{abstract}
The adaptive algorithm has been broadly used in the digital signal processing like channel estimation, channel equalization, noise cancellation, and so on. One of the most significant adaptive algorithms is the NLMS algorithm .This paper discuss about several objective optimization approaches to fast blind channel equalization. Different filter structures are presented to nullify the diverse form of noises. In this paper the proposed algorithm constrained stability least mean square (CSLMS) algorithm has distinct feature of minimizing mean square error (MSE) effectively. Different adaptive filter methods are proposed and finally CSLMS based adaptive filter algorithm is found to be the best in performance in terms of signal to noise ratio(SNR) and mean square error without increasing the computational complexity.
\end{abstract}

Keywords-Least mean square (LMS), Normalized Least mean square (NLMS), Constant modulus algorithm (CMA), constrained stability least mean square (CSLMS).

\section{Introduction}

There are many digital signal processing applications in which second order statistics cannot be specified. Such application includes channel equalization echo cancellation and noise cancellation. In these applications, filters with adjustable coefficients called Adaptive filters[1] are employed. An adaptive filter is a filter that self adjusts its transfer function according to an optimizing algorithm. It adapts the performance based on the input signal. Such filters incorporate algorithms that allow the filter coefficients to adapt to the signal statics. There are different approaches used in adaptive filtering, which are as follows:

Adaptive techniques use algorithms, which enable the adaptive filter to adjust its parameters to produce an output that matches the output of an unknown system. This algorithm employs an individual convergence factor that is updated for each adaptive filter coefficient at each iteration.

Blind equalization has the potential to improve the efficiency of communication systems by eliminating training signals. Difficulties of its application in wireless communications, however, are due largely to the characteristics of the propagation media multipath delays and fast fading. The challenge is achieving blind equalization using only a limited amount of data.

Assoc Prof, Kakatiya Institute of Technology \& Science(KITSW) India

Director, Vaagdevi College of Engineering India
The key idea of this paper is to combine the approach based on minimizing the constant modulus cost and that based on matching the second-order cyclostationary statistics. The main feature of the proposed approach is the improved convergence property over the standard CMA equalization and the improved robustness for ill-conditioned channels.

\section{Blind Channel Equalization}

The field of blind channel equalization has been in existence for over a decade now. Research has focused on developing new algorithms and formulating a theoretical justification for these algorithms. Blind channel equalization is also called as a self-recovering equalization. The aim of blind equalization is to recover the unknown input sequence to the unknown channel based solely on the probabilistic and statistical properties of the input sequence. The receiver can coordinate to the received signal and to adjust the equalizer without the reference sequence. The term blind is used in this equalizer because it performs the equalization on the data without a reference signal. Instead, the blind equalizer depends on knowledge of the signal structure and its statistics to perform the equalization. A natural question for direct adaptive equalization with reference is, "How can we adapt our filter F, without use of a reference signal". There has been extensive research on this subject for single user applications as well as multi-user applications. The Constant Modulus Algorithm is an algorithm employed for the blind adaptation problem. Fractional spaced CMA used to directly estimate equalizer $f$. It is similar to CMA. In fractional space it is global convergences.

$$
\min J=E\left[\left(\left|f^{H} X(n)\right|^{2}-R_{Z}\right)^{2}\right]
$$

update rule:

$$
f_{n+1}=f_{n}-\mu E\left(\left|f^{H} X(n)\right|^{2}-R_{Z}\right) X(n) X^{H}(n) f_{n}
$$

Algorithm:

1. Construct the received the sample

2. Construct the sample vector(n);

3. For $n=1,2 \ldots \ldots$

update function

4. Check the SER. 


\section{Constant modulus Algorithm (CMA)}

The CMA, proposed by Godard, is the most popular technique for blind equalization. Consider the base band model of a digital communication channel characterized by finite impulse response (FIR) filter and additive white Gaussian noise. In order to remove effect of channel distortion, we use the equalizer to eliminate this effect. The cost function of CMA is defined as:

$$
D_{k}^{(2)}=E\left[\left(\left|x_{k}\right|^{2}-R_{2}\right)^{2}\right]
$$

In this equation $R_{2}$ is reference signal depending on statistical properties of input signal $x_{k}$. It is defined as:

$$
R_{2}=\frac{E\left[\left|a_{k}\right|^{4}\right]}{E\left[\left|a_{k}\right|^{2}\right]}
$$

Using a stochastic gradient algorithm like LMS, we obtain updated equation of CMA:

$$
C(k+1)=C(k)-\lambda X^{*}(k) y_{k}\left(\left|y_{k}\right|^{2}-R_{2}\right)
$$

Where $\lambda$ is the step-size parameter and the asterisk denotes complex conjugation. Error signal of CMA is:

$$
e_{k}=y_{k}\left(\left|y_{k}\right|^{2}-R_{2}\right)
$$

For CMA update equation (5), assuming $e_{k}=0$ at perfect equalization, we have $\left|y_{k}\right|^{2}-R_{2}=0$.It means CMA attempts to make the equalizer output lie on the circle with radius $\sqrt{R_{2}}$. Since the cost function is based only on the equalizer output modulus, so CMA is the phase blind algorithm. Furthermore, if the frequency offset exists in equalizer output, the output constellation will spin. Godard demonstrated that the cost function can be applied even to non-constant modulus signals such as rectangular QAM constellations.

\section{Iv. Adaptive MMSE}

The signal after MMSE can be expressed in matrix form as

$$
\hat{s}(i)=w^{H} y(i)
$$

Where $y(i)=H^{T}(i) s(i)+n(i)$

$\mathrm{M}$ is the length of the MMSE Equalizer; Equalizer coefficient vector is given by

$$
w=\left[w_{1}, w_{2}, w_{3}, \ldots . w_{M}\right]^{T}
$$

The error signal e(i) is given as

$$
e(n)=d(i)-s(i)
$$

where $\mathrm{d}(\mathrm{i})$ is the desired response for MMSE Equalizer $\mathrm{d}(\mathrm{i})=\mathrm{s}(\mathrm{i}+\mathrm{D})$

$\mathrm{D}$ is the delay parameter which is usually $\mathrm{L}+1$.

The MMSE criterion is used to derive the optimal equalizer coefficients vector ' $w$ ':

$$
w=\min i \operatorname{mize} E\left\{|e|^{2}\right\}
$$

We make assumption that signal $s(i)$ and noise n(i) are independent identify distribution stochastic variable and uncorrelated to each other, then the equalizer coefficient vector $\mathrm{w}$ can be expressed as

$$
w=\left(H^{H} H+\frac{1}{S N R} I\right)^{-1} H^{H} \delta_{D}
$$

Where

$$
\delta_{D}=\left[0 \ldots \ldots .1_{D} ; 0 \ldots \ldots .0\right]_{1 X(L+M-1)}^{T}
$$

$S N R=\frac{\sigma_{s}^{2}}{\sigma_{n}^{2}}$ denotes the signal to noise ratio

I is $M \times M$ identity matrix.

To reduce the complexity caused by matrix inversion of ideal MMSE equalizer, we propose an adaptive MMSE equalizer algorithm. In code-multiplexed pilot CDMA systems, conventional adaptive equalizer is difficult to implement for lack of reference signal. The steepest descent method is used to derive adaptive equalizer algorithm in code-multiplexed pilot CDMA systems.

Hence the mean square error $\mathbf{J}$ can be expressed as

$$
J(w)=E\left[e(i) e(i)^{*}\right]=\sigma_{s}^{2}-w^{H} p-p^{H} w+w^{H} R w
$$

Where autocorrelation matrix $R=E\left[y(i) y^{H}(i)\right]$ correlation vector $p=E\left[y(i) d^{*}(i)\right], \quad \sigma_{s}^{2}$ denotes the signal power;

(.)* represents conjugate operation. Because the wireless channel is time-varying, the equalizer coefficients vector $w$ must be updated real time. Conventional adaptive algorithm requires reference signal $d(i)$, while in the downlink of code-multiplexed pilot CDMA systems, $d(i)$ is difficult to distill. To resolve this problem, the steepest decent method is used. From Eqn.8, the gradient vector is $\frac{\partial J(w)}{w}=-2 p+2 R w$ then the equalizer coefficients updating equation is

$$
w(i+1)=w(i)+2 \mu[p-R w(i)]
$$

Where parameter $\mu$ is a positive real-valued constant which controls the size of the incremental correction applied to the equalizer coefficients vector.

For the autocorrelation matrix:

$$
\begin{aligned}
& R=E\left[y(i) y^{H}(i)\right] \\
& R=E\left[s(i) s^{H}(i)\right]\left\{H^{H}(i) H(i)\right\}^{T}+E\left[n(i) n^{H}(i)\right] \\
& R=\sigma_{s}^{2}\left\{H^{H}(i) H(i)\right\}^{T}+\sigma_{n}^{2} I
\end{aligned}
$$

The cross-correlation vector 


$$
\begin{aligned}
& p=E\left[y(i) d^{*}(i)\right]=E\left[\left(H^{T}(i) s(i)+n(s)\right) s^{*}(i-D)\right] \\
& p=\sigma_{s}^{2} H^{T}(i) \delta_{D}
\end{aligned}
$$

From Eqn.9, 10, 11, we can obtain the time recursive equation of MMSE equalizer by:

$$
w(i+1)=w(i)+2 \mu \sigma_{s}^{2}\left[H^{T}(i) \delta_{D}-\left(\left\{H^{H}(i) H(i)\right\}^{T}+\frac{1}{S N R} \operatorname{Iw}(i)\right]\right.
$$

As can be seen from Eqn.15, the updating process avoids the matrix inversion operation. On the other hand, the updating process withholds the requirement to store the autocorrelation matrix $\mathbf{R}(i)$ and only the equalizer coefficients vector of last time is needed. From Eqn.15 we know, the channel convolution matrix $\mathbf{H}(i)$ is required to update the equalizer coefficients vector.

For CMA, channel response can be estimated through codemultiplexed pilot. In this paper, the low complexity slidingwindow method is used to estimate the channel coefficients, which can be expressed as

$$
\hat{\beta}_{l}(i)=\frac{1}{2 \sqrt{\alpha p w(i+1) T_{s}}} \int_{\tau_{l}\left(i+\frac{w}{2}\right) T_{s}}^{\tau_{l}\left(i+\frac{w}{2}\right) T_{s}} y(t) c_{p}^{*}\left(t-\tau_{l}\right) d t
$$

Where $\beta_{l}(i)$ is estimation of the complex gain of $l^{\text {th }}$ path; $w$ is the length of sliding-window in symbols and should be selected properly according to the varying speed of the channel.

\section{v. NLMS Algorithm}

Usually, the adaptive algorithm consists of a transfer filter for processing the input signal and an algorithm unit for update the transfer filter's coefficients, $x(n)$ the input signal; $w(n)=\left[w_{0}, w_{1}, w_{2}, \ldots . w_{l}\right]$ is the vector transfer filter coefficient; $d(n)$ is the desired function of the transfer filter; $y(n)$ is the output of transfer function $e(n)$ is the error value, and it can be written as:

$$
e(n)=d(n)-y(n)
$$

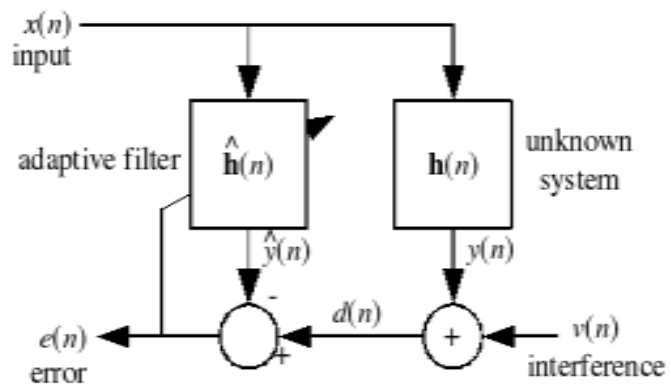

The Adaptive algorithm unit represents some algorithm to update the coefficients of the transfer filter. For LMS algorithm, the method to update the coefficients of the transfer filter is given as follows:

$$
w(n)=w(n+1)+\mu^{*} x(n) * e(n)
$$

$\mu$, is the step of LMS Algorithm.

The main drawback of the "pure" LMS algorithm is that it is sensitive to the scaling of its input $x(n)$. This makes it very hard (if not impossible) to choose a learning rate $\mu$ that guarantees stability of the algorithm. The Normalized least mean squares filter (NLMS) is a variant of the LMS algorithm that solves this problem by normalizing with the power of the input. The NLMS algorithm can be summarized as:

Parameters:

$p$ :filter order,

$\mu$ : step size Initialization

$\hat{h}(0)=0$

Computation: For $n=0,1,2 \ldots$

$$
\begin{aligned}
X(\mathrm{n}) & =[x(n), x(n-1), \ldots . . x(n-p+1)]^{T} \\
e(n) & =d(n)-\hat{h}(\mathrm{n}) \mathrm{X}(\mathrm{n}) \\
\hat{\mathrm{h}}(\mathrm{n}+1) & =\hat{h}(\mathrm{n})+\frac{\mu e^{*}(n) X(\mathrm{n})}{X^{H}(\mathrm{n}) \mathrm{X}(\mathrm{n})}
\end{aligned}
$$

The major drawback of LMS and NLMS algorithms are the large value of mean square error which results distortion in the enhanced output signal. In the CSLMS algorithm the step size parameter is inversely proportional to the squared norm of the difference between the two consecutive inputs rather than the input vector as in NLMS algorithm.

The weight update relation of the CSLMS is as follows

$$
w(n+1)=w(n)+\mu\left[\frac{\delta_{x}(n) \delta_{e}(n)}{\left\|\delta_{x}(n)\right\|^{2}}\right]
$$

\section{vi. Results and Analysis}

The performance analysis of Adaptive MMSE and adaptive CMA algorithms through CSLMS algorithm are experimentally shown in figures from 1-4.The Performance of the channel estimation is analyzed in terms of transmitted and receiver bits, Equalizers are very helpful In achieving convergence of CSLMS algorithm.

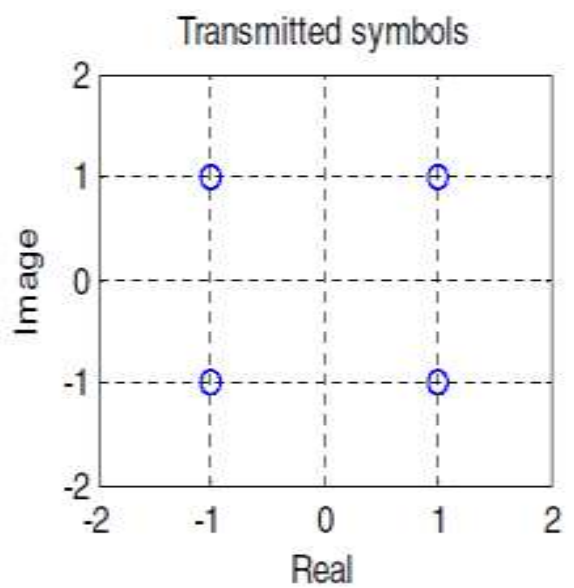


Proc. of The Third Intl. Conf. on Advances in Computing, Control and Networking - ACCN 2015.

Copyright (c) Institute of Research Engineers and Doctors, USA .All rights reserved.

ISBN: 978-1-63248-082-8 doi: 10.15224/ 978-1-63248-082-8-11

Fig.1.Transmitter symbols of Fractional-CMA

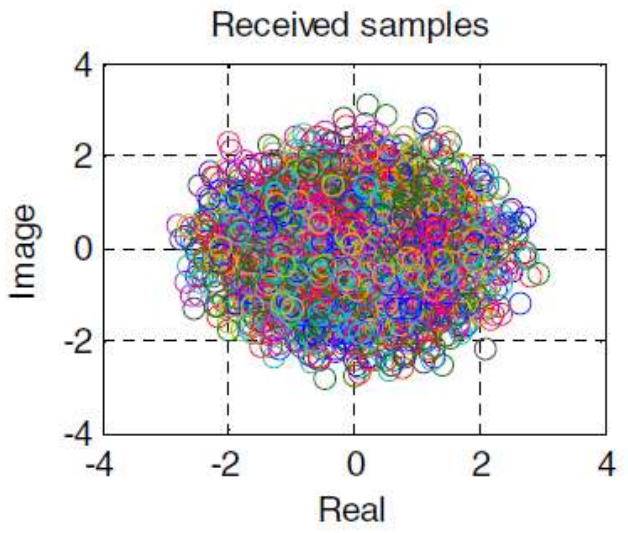

Fig.2: Receiver sample

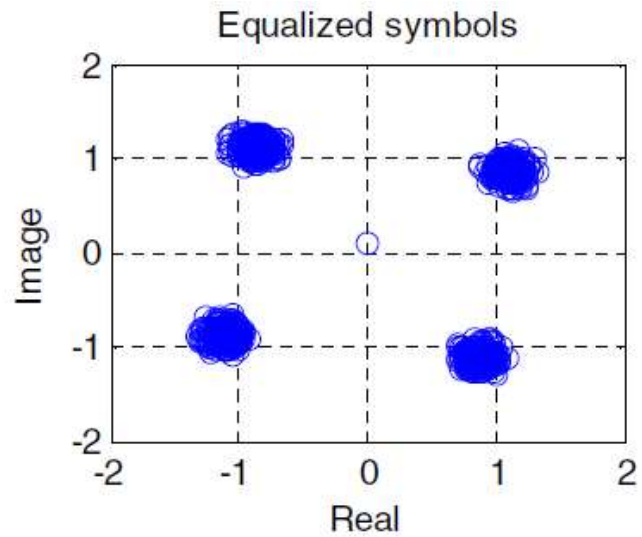

Fig..3. Adaptive Fractional- CMA Equalizer

Real

Convergence

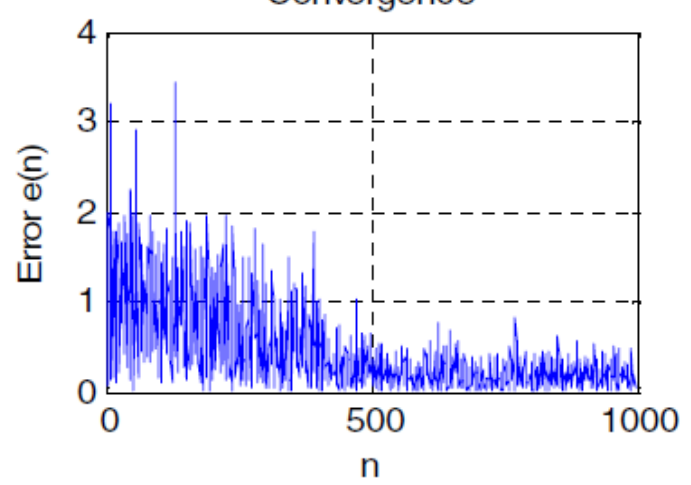

Fig.4: Adaptive MMSE Equalizer through CSLMS

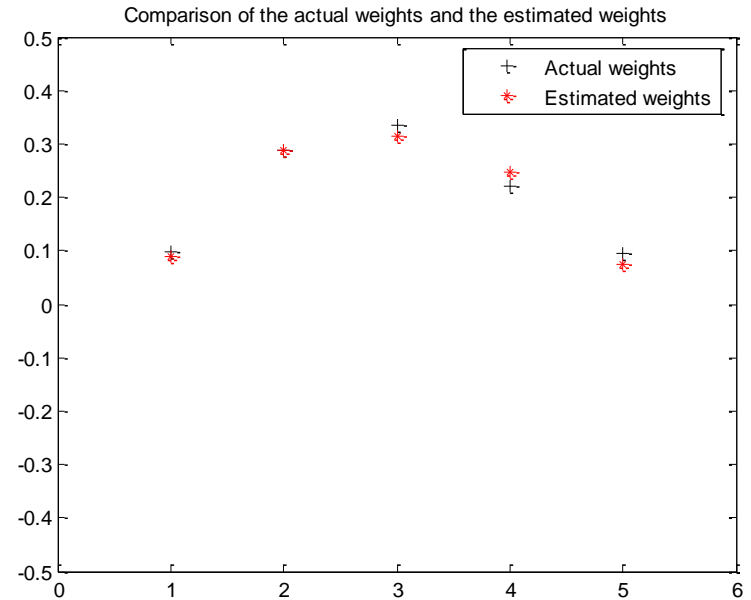

Fig..5: Comparison of the actual weight and estimated weight

The figures from 1- 3 are obtained for Adaptive CMA Equalizer, shows more efficient for equalization and convergences. from figure 4-5 are obtained for an Adaptive MMSE equalizer through CSLMS algorithm. It is found that its computational complexity is reduced, converges fast and most suitable for advanced communication systems.

\section{Conclusion}

It concludes that CSLMS algorithm shows improved performance in terms of SNR and at the same time reducing the mean square error and the misadjustment compared to the conventional methods such as LMS and normalized LMS algorithms. The simulation result shows that the performance of CSLMS algorithm is better than the NLMS algorithm

\section{References}

[1] John G. Proakis, "Digital Signal Processing Principles, Algorithms and Applications", Pearson Prentice Hall, fourth Edition, page No. 909-911. John G. Proakis, "Digital Signal Processing Principles, Algorithms and Applications", Pearson Prentice Hall, fourth Edition, page No. 909-911.

[2] Simon S. Haykin, Bernard Widrow (Editor): Least-Mean-Square Adaptive Filters, Wiley, 2003, ISBN 0-471-21570-8.

[3] Raj Kumar Thenua and S.K. AGARWAL" Simulation and Performance Analyasis of Adaptive Filter in Noise Cancellation" International Journal of Engineering Science and Technology Vol. 2(9), 2010, 4373-4378.

[4] Tandon, A.; Ahmad, M.O.; Swamy, M.N.S.; "An efficient, lowcomplexity, normalized LMS algorithm for echo cancellation", IEEE workshop on Circuits and Systems, 2004. NEWCAS 2004, pp. 161164, June 2004.

[5] Soria, E.; Calpe, J.; Chambers, J.; Martinez, M.; Camps, G.; Guerrero, J.D.M.; "A novel approach to introducing adaptive filters based on the LMS algorithm and its variants", IEEE Transactions, vol. 47, pp. 127133, Feb 2008.

[6] C. R. Johnson, Jr. et al., "Blind equalization using the constant modulus criterion: A review," Proc. IEEE, vol. 86, pp. 1927-1949, Oct. 1998.

[7] Y. Li and Z. Ding, "Global convergence of fractionally spaced Godard (CMA) adaptive equalizers," IEEE Trans. Signal Processing, vol. 44, pp. 818-826, Apr. 1996. 
Proc. of The Third Intl. Conf. on Advances in Computing, Control and Networking - ACCN 2015.

Copyright (C) Institute of Research Engineers and Doctors, USA .All rights reserved.

ISBN: 978-1-63248-082-8 doi: 10.15224/ 978-1-63248-082-8-11

[8] I. Fijalkow, A. Touzni, and J. R. Treichler, "Fractionally spaced equalization using CMA: Robustness to channel noise and lack of disparity," IEEE Trans. Signal Processing, vol. 45, pp. 56-66, Jan. 1997.

[9] W. Chung and J. P. LeBlanc, "Fractionally spaced CMA blind equalizer cost function in the presence of channel noise," in Proc. Int. Conf.Acoustics, Speech, and Signal Processing (ICASSP98), May 1998, pp.3345-3348.

About Author (s):

\begin{tabular}{|l|l|}
\hline & $\begin{array}{l}\text { R.Nirmala Devi,is currently Associate Professor, EIE } \\
\text { Dept,KITS Warangal, Research Scholar from } \\
\text { JNTUH.She Completed her B.Tech from KITSW, } \\
\text { M.Tech from NITW. Her Area of Interest are Signal } \\
\text { Processing applications ,VLSI, Wireless } \\
\text { Communications, and Image Processing.She is a Life } \\
\text { time member of IEEE,ISTE,IETE. }\end{array}$ \\
\hline
\end{tabular}

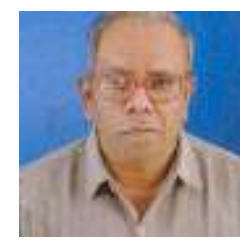

Prof. K.Kishan Rao is currently a Professor in Electronics and Communication Engineering and working as Director in Viswambhara Educational Society. He received his B.E. and M.E. degrees from Osmania University in 1965 and 1967.He is awarded with Ph.D. degree from Indian Institute of Technology, Kanpur [IIT] in 1973.He worked as Principal for National Institute of Technology, Warangal and Kakathiya Institute of Technology and Science, Warangal. He a senior member of professional bodies like IEEE [comsoc], ISTE, and IETE. He has published over 78 International articles. He currently serves as Editor for International Journal of Wireless Personal communication, Springer and International Journal of Wireless Networks, Springer. He guided 08 Ph.D scholars and guided 62 Master Projects. His research interests are in the areas of Wireless Communications, Signal Processing Applications and Cooperative Mobile Communications. 